\title{
PRIMARY TUBERCULOSIS OF THE GUM
}

\author{
BY \\ T. A. BRAND and C. F. BALLARD \\ From the Paediatric Department, St. George's Hospital, London
}

(Received for Publication May 26, 1950)

Apart from the three cases of primary tuberculous ulceration of the mouth, with involvement of the relevant submaxillary gland, recently reported (January 23, 1950) by Boyes and Miller at a meeting of the Odontological Section of the Royal Society of Medicine but not yet published in the Proceedings of the Society, no record has been found of cases in this country or in the United States of America. A case in this country, therefore, is sufficiently uncommon to merit recording. There was no response to treatment locally but, following the exhibition of streptomycin systemically, the result has been most satisfactory, although it is too early to claim a cure.

In the last 17 years there have been a number of cases published in European countries, amongst which the following may be mentioned: Akerberg (1933), Vallette and Rosenkranz (1936), Quintero (1947), Bernheim and Confavreux (1948), Lebourg and Roy (1949), Raison, Lepoivre, and Chalvet (1949).

\section{Case Report}

The case we report is that of a girl, A.J., aged 5 years and 10 months, who was referred to the out-patient department by her doctor because of general malaise for the last few months, associated with gingivitis affecting particularly the lower gums, and a chronic ulcer of the lower sulcus with enlarged submaxillary and cervical glands. The ulcer was known to have been present for eight weeks and was treated locally with penicillin lozenges and generally by a 'tonic' and vitamin $C$. At the onset some fever was noted. The patient had a habit of sucking the first finger of the right hand which, during the act, rested against the lower right incisor. In view of the contact described below this habit may have been the means of introducing the infection.

Previous History. Delivery had been normal at full term, and the birth weight was $7 \frac{1}{2} \mathrm{lb}$. The baby was breast-fed for nine months. The milestones had been normal.

At 1 year she had whooping cough, and at 4 years, measles. She always had a poor appetite and a tendency to dental caries.

In February, 1949, she had two teeth extracted followed by high fever and ' $a$ chill on the bladder'. A tuberculin jelly patch test at this time is reported as having been negative. The test was carried out as the child's doctor wished to exclude abdominal tuberculosis. On recovery the patient remained well until the present episode.

Family History. The mother, father and sister (aged 2 years 11 months) are well. Radiological examination of their chests has revealed no evidence of tuberculosis. The grandmother and great-uncle were tuberculous, but there has been no contact.

Before the oral condition developed the patient had visited a neighbour on a number of occasions. This person is now known to have been suffering from pulmonary tuberculosis, and has since been admitted to hospital for treatment.

The patient had had only pasteurized milk and this had always been brought to the boil before use.

Examination. She was a pale child with evidence of loss of weight (393 lb., i.e. $18 \cdot 1 \mathrm{~kg}$.). Her skin was moist. A painless indolent ulcer, oval in shape, extended from the right lower deciduous canine tooth mesially to the right lower permanent central and from the gingival margin to the base of the sulcus. In the middle of this area there was a flake of hard tissue, probably a piece of necrotic bone in process of separation. This was confirmed later by biopsy. There was gingivitis involving the whole lower gum and enlargement of the submaxillary gland, which was firm, smooth and rounded, an inch $(2.5 \mathrm{~cm}$.) in diameter and painless. The nose and throat were healthy, and there was no abnormality in the respiratory, cardiovascular, and central nervous systems, and abdomen.

Because a chronic ulceration of such duration in a child is very unusual the patient was referred to the Eastman Dental Clinic, where a blood count gave haemoglobin $82 \%$, white cells 14,200 per c.mm. (polymorphs $45 \%$, lymphocytes $52 \%$, eosinophils $2 \%$, and monocytes $1 \%$ ).

Biopsy was performed twice, the first being inconclusive, the second showing 'granulation tissue in which there are several small histological tubercles, with early caseation. Occasional acid-alcohol-fast bacilli were seen in tissues (Figs. 1-3).

A positive culture was obtained at the end of five weeks. A guinea-pig was inoculated, and in six weeks showed lesions typical of tuberculosis.

In view of the biopsy report, the patient was admitted to the Victoria Hospital for Children, Tite Street, on September 29, 1949. Both the local and general conditions were unchanged from those reported above. 


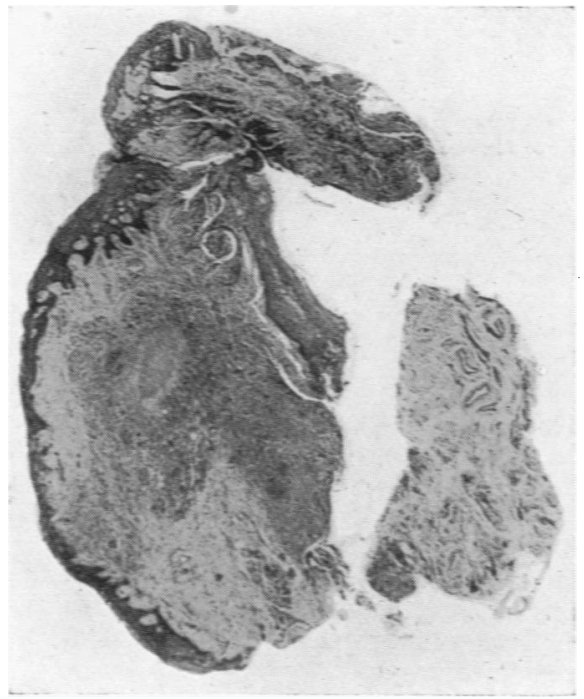

Fig. 1.-Photograph of biopsy specimen showing area of caseation surrounded by giant cell systems.

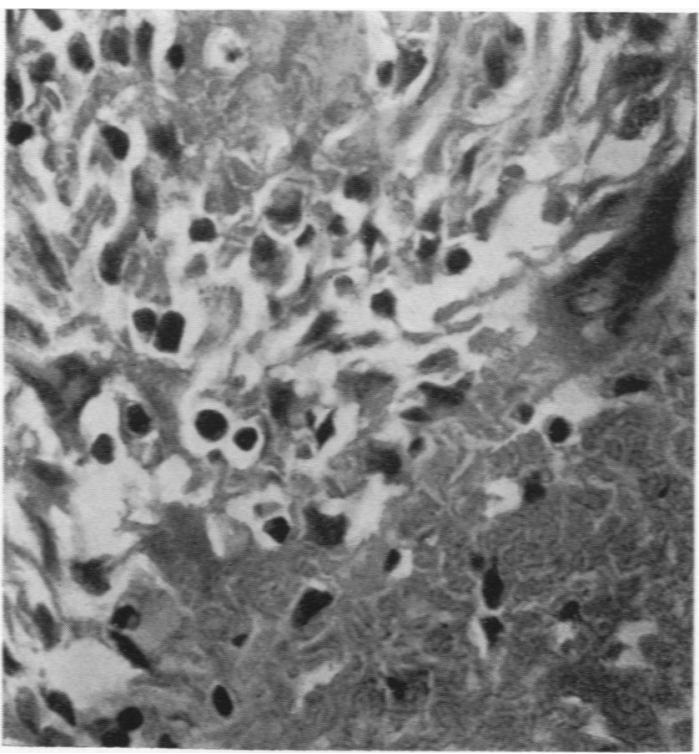

Fig. 3.-High power view to show margin of area of caseation necrosis with adjacent epithelioid cells and giant cells. 
The following further investigations were carried out. The Mantoux test, 1:1,000, was strongly positive. No bony abnormality of the jaw was seen radiologically, nor was any abnormality shown in the thorax and abdomen.

A second blood count gave haemoglobin $80^{\circ}$, W.B.C. 14,300 per c.mm. (polymorphs $62 \%$, lymphocytes $33 \%$, monocytes $3 \%$, eosinophils $1 \%$, and Turck, $1_{o}^{\circ}$ ). The E.S.R. was $14 \mathrm{~mm}$. in 1 hour (micro.).

Progress and Treatment. The temperature remained normal during the ten weeks' stay in hospital except for an occasional rise to $9^{\circ}$ in the evening. Pulse and respirations were normal throughout.

The patient was kept on bed rest, and the affected area was cleansed with sterile cotton wool. No antiseptics were used.

On October 10, as there was no improvement, streptomycin calcium complex (Glaxo), 0.38 g., b.d.s., intramuscularly, was given for three weeks, the dose being based on the Medical Research Council recommendations of $0.02 \mathrm{~g}$. per lb. body weight per day. The total dose in this course was $17.48 \mathrm{~g}$.

On October 27 the course of streptomycin was completed and the ulcer was almost healed. The gingivitis and general adenitis had subsided. The submaxillary gland had markedly decreased in size and the general health was much improved. The jaw was again examined radiologically and no bony abnormality found.

By November 10 the ulcer was healed, and the gland firm and further reduced in size. Weight gain continued.

There was slight but persistent inflammation round the second lower right deciduous incisor tooth which was found to be loose. It was uncertain whether this looseness was due to natural causes or a sequel to the previous inflammatory condition. It was decided to extract this and two other carious teeth under a streptomycin umbrella. Intramuscular streptomycin, $0.38 \mathrm{~g}$. twice daily, was given for ten days (total $7 \cdot 6$ g.). After the extractions the gums healed satisfactorily and the patient was discharged.

Early in December (December 7,1949$)$ the child was in good general health (weight $42 \mathrm{lb}$., i.e. $19.0 \mathrm{~kg}$.) : the mouth was healthy and the ulcer remained healed. On the last visit to the out-patient department (January i 7, 1950) there had been a further gain in weight of 2 lb. $(0.906 \mathrm{~kg}$.), the progress in general health continued, and the mouth was healthy. The right submaxillary gland was now hard, $1 \mathrm{~cm}$. in diameter, and the remaining glands of the neck just palpable with some small shotty glands in both posterior triangles.

Locally the most notable fact was the painless nature of the ulcer and glands. Before streptomycin the ulcer remained in an indolent state, showing no response to local treatment or to vitamins D (approximately 1,200 units daily) and $A$, and vitamin $C$ by mouth. On investigation, the tuberculous nature of the lesion was proved and no other cause of the patient's general malaise could be found. There was no evidence of a primary tuberculous infection elsewhere.

\section{Discussion}

From the literature that has been reviewed, the following observations are interesting.

The cases reported occurred between the ages of 7 and 12 years. They were associated with the loss of the deciduous teeth, or eruption of the permanent teeth in the cases of Akerberg (1933), Schmiziger (1945), Quintero (1947), Lebourg and Roy (1949), and Raison, Lepoivre, and Chalvet in the same year.

Characteristically there has been found an indolent ulcer of the gum, resistant to local treatment, painless to touch and with enlarged relevant glands with little tenderness on palpation. This latter finding is in contradistinction to the tenderness of glands associated with other infections of the oral cavity (Vallette and Rosenkranz, 1936; Schmiziger, 1945; Quintero, 1947; Bernheim and Confavreux, 1948; Lebourg and Roy, 1949; and Raison, Lepoivre, and Chalvet (1949).

Biopsy of an ulcer was performed by Akerberg (1933) when typical tubercle formations were demonstrated. These were shown in the case we report, and in addition, acid-alcohol-fast bacilli were found. Tubercle bacilli have been recovered from swabs taken from the ulcers (Bernheim and Confavreux, 1948, and Raison, Lepoivre, and Chalvet, 1949).

Two cases (Quintero, 1947; and Lebourg and Roy, 1949), ended fatally, the first with evidence of both miliary tuberculosis and tuberculous meningitis, and the second with tuberculous meningitis only. In the discussions following the reporting of Lebourg and Roy's case, Bataille (1949) stated that he had found records in the literature of 16 cases of primary tuberculosis of the gum, and in five of these tuberculous meningitis had followed with fatal results. Lebourg and Roy, commenting on this stated that they had had 14 cases in the Trousseau Hospital, Paris, and in only one, that reported above, had meningitis followed.

Akerberg (1933) pointed out that the glands appear to be enlarged beyond that expected from the indolent nature of the ulcer of the gum. They commented on the tendency of the glandular enlargement in tuberculous infections to be much greated in primary than in secondary infections.

In a number of instances it has been suggested that the infection is acquired from milk. It is often associated with local trauma of the gum due to the loss of the deciduous teeth or eruption of the permanent teeth (Akerberg, 1933; Schmiziger, 1945; Bernheim and Confavreux, 1948; and Raison, Lepoivre, and Chalvert, 1949). The evidence can only be regarded as circumstantial in the cases quoted. Proof of the recent exposure to infected milk was shown. but in no instance was it established 
that the lesion was due to the bovine tubercle bacillus.

In cases where radiological examination of the jaw was carried out no evidence of bony involvement was demonstrated (Vallette and Rosenkranz, 1936; Quintero, 1947; and Raison, Lepoivre, and Chalvet 1949). The case we describe was examined twice, but on each occasion there were no radiological changes to be seen in the underlying bone, although initially the alveolar bone was involved as proved by the biopsy.

In one case (Vallette and Rosenkranz, 1936) within six weeks of the primary infection of the gum being observed the patient developed erythema nodosum.

In the literature reviewed no evidence was advanced of a preceding contact with a case of tuberculosis, although it is not possible to judge from the reports how thoroughly this aspect was investigated in every instance. In the case we describe there was a possible human source of infection.

It is of interest to note that oral tuberculous infection, secondary to a pulmonary lesion, is relatively common and well recognized. Much has been written on this subject, and the paper of Darlington and Salman (1937) provides a comprehensive review of the forms in which it may occur.

Though it is clear that primary tuberculous ulceration of the gum is not a frequent occurrence, the fact that cases have been known to terminate fatally following a generalized spread makes it a condition worthy of note. Since most cases appear to follow a relatively benign course and heal in a way comparable to the majority of primary tuberculous infections elsewhere in the body, it is possible that the condition may occur more often than is recognized, especially in rural areas where milk may not be pasteurized. Perhaps, in some instances, it may be masked by a local secondary infection so that the underlying tuberculous lesion is not recognized.

\section{Summary}

A case is described of primary tuberculosis of the gum which responded satisfactorily to streptomycin treatment. The literature is reviewed.

Our thanks are due to Dr. Charles Pinckney for his permission to report this case and for his encouragement and advice in its preparation. We wish to thank Professor R. A. Webb and Drs. M. P. Jevons and I. P. Beswick for the report on the biopsy and subsequent identification of the tubercle bacillus. We also thank Professor John Boyes and Dr. F. J. W. Miller for permission to quote their cases before they appear in the Proceedings of the Royal Society of Medicine.

REFERENCES
Åkerberg, E. (1933). Acta derm.-venereol., Stockh., 14, 127.

Bataille, R. (1949). Rev. Stomatol., Paris, 50, 368.

Bernheim, M., and Confavreux, J. (1948). Sem. Hôp. Paris, 24, 1206.

Darlington, C. C., and Salman, I. (1937). Amer. Rev. Tuberc., 35, 147.

Lebourg, L., and Roy, M. (1949). Rev. Stomatol., Paris, $50,364$.

Quintero, J. (1947). Ibid., 48, 417.

Raison, Lepoivre, and Chalvet (1949). Ibid., 50, 362.

Schmiziger, P. (1945). French Summary by C. Bonsack. Schweiz. Mschr. Zahnheilk., p. 1,087.

Vallette, A., and Rosenkranz, B. (1936). Bull. Soc. Pédiat., Paris, 34, 465. 